\title{
Design of Ultra-Low Power Pulse-Driven MEMS Oscillator
}

\author{
Seonho Seok ${ }^{1}$, Giorgio Cristiano ${ }^{2}$, and Taekwang Jang ${ }^{2}$ \\ Center for Nanoscience and Nanotechnology (C2N), University-Paris-Saclay, Orsay, France ${ }^{1}$ \\ ETH Zürich, Zurich, Switzerland ${ }^{2}$ \\ Email: seonho.seok@u-psud.fr
}

\begin{abstract}
This paper presents an energy efficient pulse-driven on-chip MEMS oscillator which consists of MEMS resonator and sustaining CMOS circuit. Conventional oscillators are driven by means of an inverter-based amplifier low-pass filtered through a series resistor. However, this architecture suffers from multiple energy losses. Firstly, the inverter amplifier is driven by a sinusoidal input, thus consuming significant static power. Additional power is also consumed by the series resistance. The proposed pulsed driver provides a $\mathbf{1 8 0}^{\circ}$ degree shift, effectively replacing the amplifier. The driver injects energy in the oscillator by periodically switching one oscillator end to $V_{\text {DD }}$ when the other end reaches the bottom voltage, and to $V_{S s}$ when the other end reaches the peak voltage. The proposed configuration reduces the static power consumption, as the switch is driven by two rectangular voltages and reduces leakage by activating only one of the two switches at a time. This pulse-driven oscillator, running at $33.14 \mathrm{kHz}$, is simulated to consume $835 \mathrm{pW}$ when driven at $0.8 \mathrm{~V}$, leading to more than $x 15$ power saving with respect to the traditional configuration, which consumes $13.2 \mathrm{nW}$.
\end{abstract}

Keywords-Low power, pulse-driven, MEMS oscillator

\section{INTRODUCTION}

The Internet of things (IoT) is a system of interrelated computing devices being able to transfer data over a network without requiring human-to-human or human-to-computer interaction. Miniaturization and interactive communication are the two main topics that dominate the recent research in the internet-of-things (IoT) [1-5]. The next generation of computing platforms, which is the IoT, increases proximity to the source of the information rather than to humans requesting much more aggressive miniaturization. The advantages of miniaturization are lowering cost, space reduction, and enhanced mobility. To this sense, mm-scale computer has been recently reported by university of Michigan for energy efficient sensor node for new IoT application [6]. All the functional ICs of the mm-scale computer have been designed to be operational only with the energy harvested photovoltaic cell integrated with the mm-scale computer. From technological point of view, the mm-scale computer has been implemented by stacking of multiple Si chips. Chip stacking has been done by die attach with gluing and wire bonding. As wire bonding has been used, epoxy overmolding should be followed to finalize the die stacking packaging. To improve technological limit of precision of the conventional packaging processes such as wirebonding, flip-chip bonding for miniaturization of IoT system, a novel packaging and heterogeneous integration method is highly required. Sensor node for IoT needs an essential element for synchronization between computing devices, which is socalled timer. For timekeeping application, $32 \mathrm{kHz}$ quartz tuning fork crystals and oscillators are mostly used. Key specifications for this clock are frequency accuracy, PCB area and power consumption. Size reduction rate of $32 \mathrm{kHz}$ quartz oscillator tends to be slowing due to scaling limitations and packaging/assembling difficulties and thus MEMS-based oscillators matching or outperforming the quartz counterparts have been introduced in the past decade. Indeed, MEMS-based oscillators have merits of reduced size as they are fabricated by semiconductor process. However, MEMS resonator and ICs are still being packaged by using conventional wire-bonding or flip-chip bonding technique in 2D or 3D [7]. Advanced packaging technique such as FOWLP (Fan-Out Wafer Level Package) could provide much lower packaging profile which is essential for miniaturized IoT systems.

In this paper, MEMS resonator and pulse-driver are designed for low-power timekeeper. MEMS resonator design and pulsedriven MEMS oscillator design are explained in section 2 and section 3 respectively. Section 4 will conclude our work.

\section{MEMS RESONATOR DESIGN}

MEMS resonator in flexural-mode and its equivalent massspring-damper model are shown in Fig. 1. Governing equation of the MEMS resonator is given as below.

$$
m_{e f f} \frac{\partial x^{2}}{\partial t^{2}}+c_{e f f} \frac{\partial x}{\partial t}+k_{e f f} x=F_{e}(t)
$$

,where $m_{\text {eff }}$ is effective modal mass, $c_{\text {eff }}$ is effective damping coefficient, and $\mathrm{k}_{\text {eff }}$ is effective stiffness.

The effective modal mass is designed through ANSYS modal analysis as shown in Fig. 2 and the effective stiffness and damping coefficient are determined with following equations.

$$
\begin{gathered}
k_{e f f}=\omega_{r}^{2} \times m_{e f f} \\
c_{e f f}=\frac{\omega_{r} m_{e f f}}{Q}
\end{gathered}
$$

,where $\omega_{\mathrm{r}}$ is angular resonant frequency.

Electrostatic force $F_{e}(t)$ is given by

$$
F_{e}(t)=\eta_{e} v_{a c}(t)
$$




$$
\eta_{e}=V_{P} \frac{\varepsilon_{0} A}{g_{0}^{2}}
$$

,where $\eta_{\mathrm{e}}$ is electromechanical coupling factor.

Equivalent electrical circuit parameters can be found as follows.

$$
L_{x}=\frac{m_{e f f}}{\eta_{e}^{2}} \quad C_{x}=\frac{\eta_{e}^{2}}{k_{e f f}} \quad R_{x}=\frac{c_{e f f}}{\eta_{e}^{2}}
$$

Design parameters of the MEMS resonator are summarized in Table. 1 and the equivalent electrical circuit model is shown in Fig. 3. Note that $\mathrm{Cp}$ is feedthrough capacitance between driving and sensing electrodes and $\mathrm{Co} 1$ and $\mathrm{Co} 2$ are parasitic capacitance related with driving and sensing pads. If mass is properly grounded, no capacitive coupling exists between driving and sensing electrodes.

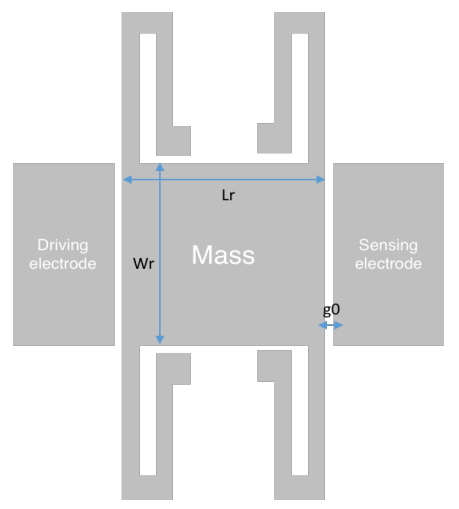

(a)

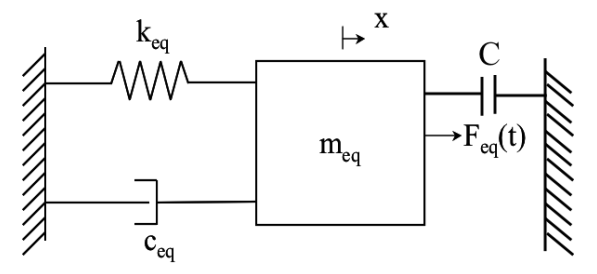

(b)

Fig. 1 MEMS resonator and its equivalent model with electrostatic transduction

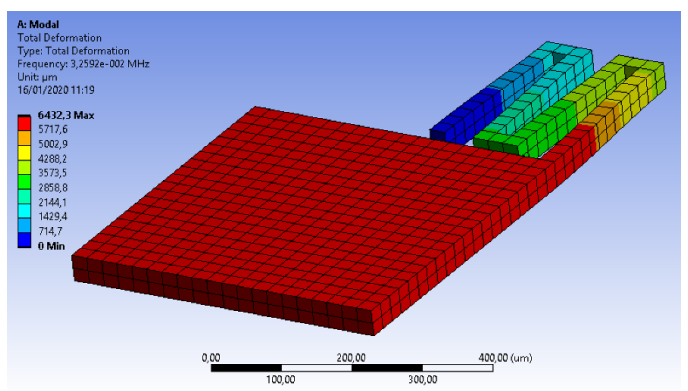

Fig. 2 ANSYS modal analysis result: $1^{\text {st }}$ mode

\begin{tabular}{|c|c|c|c|}
\hline \multicolumn{2}{|c|}{ Resonator } & \multicolumn{2}{|c|}{ Capacitive Transducer } \\
\hline Lr & $1000 \mu \mathrm{m}$ & $\begin{array}{c}\text { Electrode } \\
\text { width, } \\
\text { We }\end{array}$ & $1000 \mu \mathrm{m}$ \\
\hline $\mathrm{Wr}$ & $1000 \mu \mathrm{m}$ & Gap, go & $1000 \mathrm{~nm}$ \\
\hline $\begin{array}{l}\text { Effective } \\
\text { mass, meff }\end{array}$ & $10.1 \mathrm{E}-8(\mathrm{~kg})$ & VP & $30 \mathrm{~V}$ \\
\hline $\begin{array}{c}\text { Effective } \\
\text { stiffness, keff }\end{array}$ & $4238(\mathrm{~N} / \mathrm{m})$ & $\eta_{e 1}, \eta_{e 2}$ & $1.06 \mathrm{E}-5$ \\
\hline $\begin{array}{l}\text { Effective } \\
\text { damping, } \\
\text { ceff }\end{array}$ & $\begin{array}{c}4.14 \mathrm{E}-6 \\
(\mathrm{Ns} / \mathrm{m}) \\
@ \mathrm{Qnom}=50 \\
00\end{array}$ & Lx & $895 \mathrm{H}$ \\
\hline $\mathrm{fm}$ & $32.6 \mathrm{kHz}$ & $C x$ & $26.6 \mathrm{fF}$ \\
\hline Lm & $1.01 \mathrm{E}-7(\mathrm{H})$ & $\mathrm{Rx}$ & $36.7 \mathrm{kohms}$ \\
\hline $\mathrm{Cm}$ & $2.56 \mathrm{E}-4(\mathrm{~F})$ & & \\
\hline $\mathrm{Rm}$ & $\begin{array}{c}4.14 \mathrm{E}-6 \\
\text { (ohm) }\end{array}$ & & \\
\hline
\end{tabular}

Table 1: Design parameters of MEMS resonator

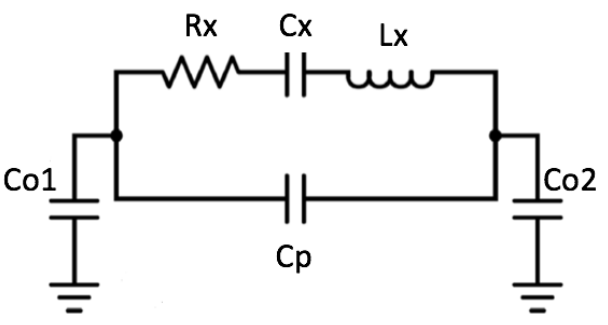

Fig. 3 Electrical model of a resonator

\section{PULSE-DRIVEN MEMS OSCILlator}

Conventional drivers for the resonator are implemented by an inverter based amplifier in series with a resistor as shown in Fig. 4. Such oscillators have critical limitations in reducing the power consumption. First, the inverter-based amplifier suffers from the short circuit current while buffering the sinusoidal signal from the resonator to a square wave to drive the series resistor $\mathrm{R}_{\mathrm{s}}$. Second, the series resistance also dissipates a significant power due to a large voltage applied to it. Therefore, it is necessary to develop a circuit technique that improves energy regeneration efficiency by reducing the energy loss in the amplifier and the regeneration resistor.

A pulsed driver, which was proposed to implement an ultralow-power crystal oscillator [8], is an effective way to improve the energy efficiency of the MEMS oscillator. Fig. 5 shows a circuit schematic of the pulsed driver based oscillator. In this circuit, a resonation voltage, $\mathrm{OSC}_{\mathrm{IN}}$, is buffered to a delaylocked loop (DLL) to generate two narrow pulses that are located at the peaks of the sinusoidal oscillation voltages, $\mathrm{OSC}_{\mathrm{IN}}$ and OSC $\mathrm{CRRV}_{\mathrm{DR}}$. Level shifters provide proper gate voltages for MN1 and MP1 to inject the energy into the resonator. As the switching transistors, MN1 and MP1, are turned on only for a 


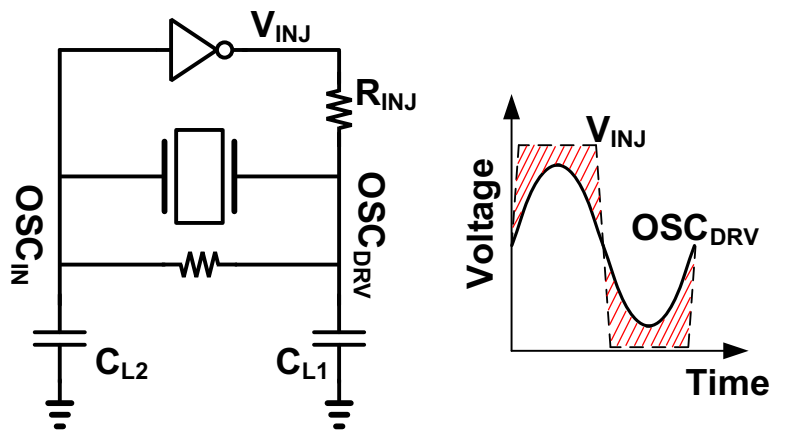

Fig 4: Conventional MEMS resonator

short duration while the oscillation voltages reach their peaks, the voltage across the switches during the energy injection is small so that the most of the energy driven from the supply is delivered to the resonator with a minimal conduction loss. Furthermore, the static power consumption of MN1 and MP1 is very low because the transistors receive sharp rectangular pulses to reduce the leakage current.

To find the theoretical limit of the power consumption of the MEMS resonator, a theoretical analysis and the simulation is performed as follows.

First, four capacitors composing the MEMS oscillator can be merged as the following equation so that the resonator model is simplified to a series LCR circuit.

$$
C_{e q}=C_{x} \|\left(C_{P}+C_{O 1} \| C_{O 2}\right)
$$

Then, by assuming an exponential settling of OSC $\mathrm{CRR}_{\mathrm{DR}}$ node voltage during the injection time, the residual voltage at the peak of the oscillation, $\Delta \mathrm{V}$, can be expressed as the following equation:

$$
\begin{aligned}
\Delta V & =\frac{V_{D D L}-V_{S S L}-V_{O S C}+\Delta V}{2}\left(1-e^{-\frac{T_{O N}}{R_{S W} C_{e q, S w}}}\right) \\
& =\frac{V_{S U P}-V_{O S C}+\Delta V}{2} \gamma
\end{aligned}
$$

Where $T_{O N}$ and $R_{S W}$ are the turn-on time and resistance of the switch driver, respectively. $C_{e q, s w}$ is the equivalent capacitance that the switch driver sees toward the resonator which is equal to $C_{O 2}+\left(C_{O 1} \| C_{P}\right)$. A settling factor, $\gamma$, is adopted to express how much exponential settling was completed while the switch is turned on.

By rearranging (2), $\Delta \mathrm{V}$ can be found as

$$
\Delta V=\tanh \left(\frac{T_{O N}}{2 R_{S W} C_{e q, s w}}\right)\left(V_{S U P}-V_{O S C}\right) .
$$

Consequently, the oscillation amplitude can be found by relating $\mathrm{V}_{\text {Sup }}$ and $\Delta \mathrm{V}$.

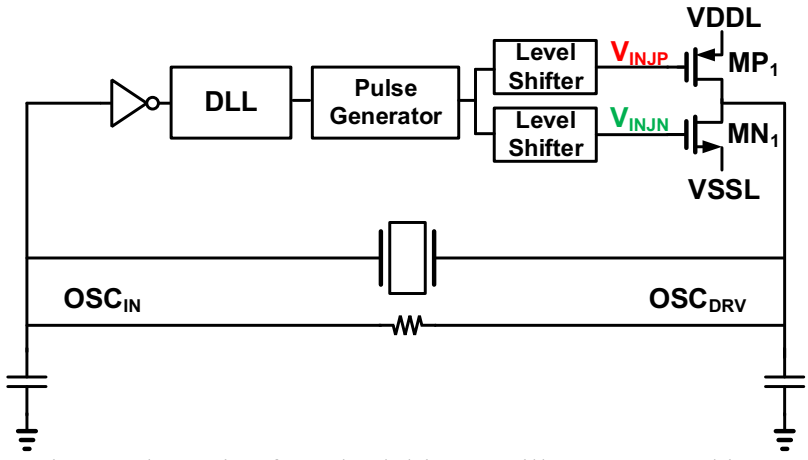

Fig 5: Schematic of a pulsed driver oscillator proposed in [8]

VDDL

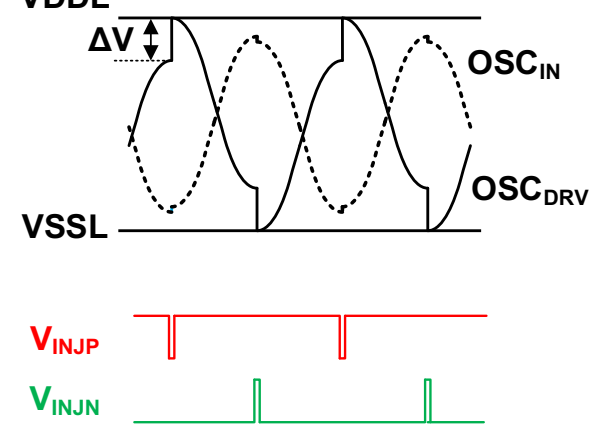

Fig 6: Waveforms of the signals in the pulsed driver based oscillator

$$
V_{O S C}=\frac{\tanh \left(\frac{T_{O N}}{2 R_{S W} C_{e q, s w}}\right) C_{e q, s w}}{\frac{\pi R_{x}\left(C_{P}+C_{O 1} \| C_{O 2}\right)^{2}}{\sqrt{L_{x} C_{e q}}}+\tanh \left(\frac{T_{O N}}{2 R_{S W} C_{e q, s w}}\right) C_{e q, s w}} V_{S U P}
$$

Note that the oscillation amplitude is inversely proportional to the switch resistance when Rsw is large as can be seen through the following approximation.

$$
\begin{aligned}
V_{O S C} \approx & \frac{C_{e q, s w} \frac{T_{O N}}{2 R_{S W} C_{e q, s w}}}{\frac{\pi R_{x}\left(C_{P}+C_{O 1} \| C_{O 2}\right)^{2}}{\sqrt{L_{X} C_{e q}}}} \\
= & \frac{\sqrt{L_{m} C_{e q}} V_{S U P}}{2 \pi R_{x}\left(C_{P}+C_{O 1} \| C_{O 2}\right)^{2}} \cdot \frac{T_{O N}}{R_{S W} C_{e q, s w}}
\end{aligned}
$$

On contrary, the oscillation amplitude reaches to its peak when Rsw becomes very small so that $\gamma$ is close to 1 .

$$
V_{O S C, \max }=\frac{C_{e q, s w}}{\frac{\pi R_{x}\left(C_{P}+C_{O 1} \| C_{O 2}\right)^{2}}{\sqrt{L_{x} C_{e q}}}+C_{e q, s w}} V_{S U P}
$$




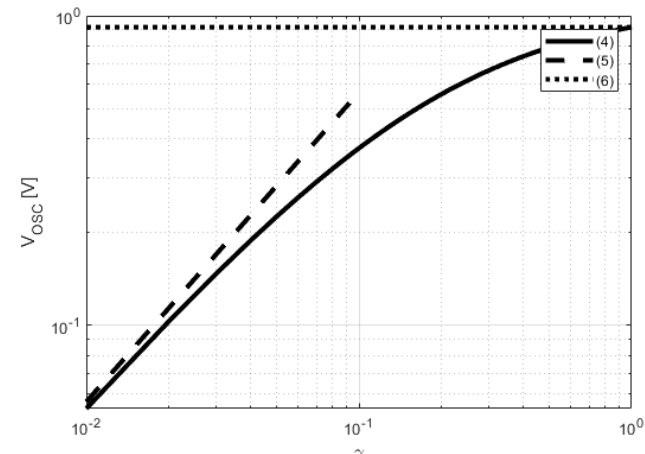

Fig 7: Simulation results of the oscillation amplitude

The oscillation amplitude in (4), (5) and (6) are plotted in Fig. 7. The discussion to this point shows that the pulsed driver oscillator's amplitude is different from a conventional oscillator model whose energy is regenerated by a trans-conductance circuit. In the conventional mode, the regeneration current amount is proportional to the oscillation amplitude, so there exists a mathematical limit in reducing the oscillation amplitude to achieve the low power. However, a pulsed driver can sustain its oscillation with an arbitrarily small oscillation amplitude as found in (4). This gives a significant advantage in achieving the low power operation as can be found in the following derivations.

The energy that needs to be regenerated by the pulsed driver can be found as the following equation:

$$
\begin{aligned}
E_{\text {reg }} & =C_{e q, s w} \cdot V_{O S C} \cdot \Delta V \\
& =\tanh \left(\frac{T_{O N}}{2 R_{S W} C_{e q, s w}}\right) C_{e q, s w} V_{O S C}\left(V_{S U P}-V_{O S C}\right)
\end{aligned}
$$

Then, the energy provided by the supply can be calculated by integrating the current during the switching operation.

$$
\begin{aligned}
E_{S U P} & =\int_{V_{S U P}} i_{S U P}(t) d t=V_{S U P} Q_{S U P} \\
& =\frac{V_{S U P}}{V_{O S C}} E_{r e g}
\end{aligned}
$$

By combining (5), (7) and (8), the energy derived from the supply can be found as the following equation when Rsw is large.

$$
E_{S U P} \approx \frac{T_{O N} V_{S U P}^{2}}{2 R_{S W}}
$$

This implies that a large Rsw reduces the oscillation amplitude by limiting the energy driven by the supply. When Rsw is small enough to achieve a full exponential settling during the energy injection period, the maximum energy is driven from the supply as the following equation:

$$
E_{S U P, \max }=\frac{\pi R_{x}\left(C_{P}+C_{O 1} \| C_{O 2}\right)^{2} C_{e q, s w}}{\pi R_{x}\left(C_{P}+C_{O 1} \| C_{O 2}\right)^{2}+C_{e q, s w} \sqrt{L_{x} C_{e q}}} V_{S U P}^{2}
$$

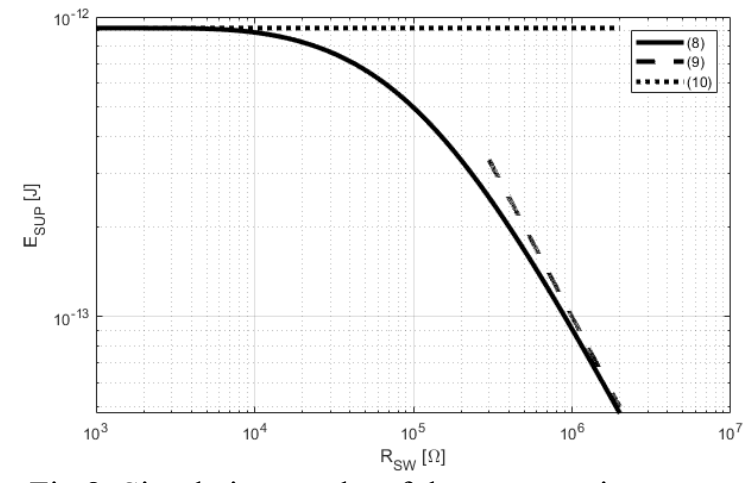

Fig 8: Simulation results of the regeneration energy

Fig. 8 shows Esup as a function of switch resistance. Esup is inversely proportional to $\mathrm{R}_{\mathrm{sw}}$ when it is large while it asymptotically approaches Esup,max when RSW becomes small. While a large $\mathrm{R}_{\mathrm{sw}}$ is advantageous in reducing the energy driven from the supply at each restoration cycle, the regeneration efficiency is higher with a small $\mathrm{R}_{\mathrm{sw}}$ as the voltage across the switch becomes smaller.

$$
\eta=\frac{E_{\text {reg }}}{E_{S U P}}=\frac{V_{O S C}}{V_{S U P}}
$$

Therefore, a given amplitude of the oscillator for its jitter and long-term stability specification, it is more advantageous to reduce the supply voltage and operate the oscillator with a small $\mathrm{R}_{\mathrm{Sw}}$ rather than including a large $\mathrm{R}_{\mathrm{Sw}}$ with a high supply voltage.

\section{CONCLUSION}

Ultra-low power MEMS oscillator for timekeeper of future IoT elements is proposed and designed. Theoretical background for low power consumption of the proposed pulsed driver based oscillator compared with inverter based amplifier is presented.

\section{REFERENCES}

[1] T. Jang et al., "Circuit and system designs of ultra-low power sensor nodes with illustration in a miniaturized GNSS logger for position tracking: Part II-Data communication, energy harvesting, power management and digital circuits," IEEE Trans. Circuits Syst. I, Reg. Papers, vol. 64, no. 9, pp. 2250-2262, Sep. 2017.

[2] S. Jeong, Z. Foo, Y. Lee, J.-Y. Sim, D. Blaauw, and D. Sylvester, “A fully-integrated $71 \mathrm{nW}$ CMOS temperature sensor for low power wireless sensor nodes," IEEE J. Solid-State Circuits, vol. 49, no. 8, pp. 1682-1693, Aug. 2014.

[3] Y. Lee et al., "A modular $1 \mathrm{~mm}$ die-stacked sensing platform with low power I2C inter-die communication and multi-modal energy harvesting," IEEE J. Solid-State Circuits, vol. 48, no. 1, pp. 229-243, Jan. 2013.

[4] G. Kim et al., "A millimeter-scale wireless imaging system with continuous motion detection and energy harvesting," in Symp. VLSI Circuits Dig. Tech. Papers, Jun. 2014, pp. 1-2.

[5] I. Lee et al., "Circuit techniques for miniaturized biomedical sensors," in Proc. IEEE Custom Integr. Circuits Conf., Sep. 2014, pp. 1-7.

[6] www.spectrum.ieee.org Millimeter-Scale Computers: Now With DeepLearning Neural Networks on Board 
[7] A.C.Fischer, et al, "Integrating MEMS and ICs," Microsystems \& Nanoengineering, 2015
[8] D. Yoon, T. Jang, D. Sylvester and D. Blaauw, "A $5.58 \mathrm{nW}$ Crystal Oscillator Using Pulsed Driver for Real-Time Clocks," in IEEE Journal of Solid-State Circuits, vol. 51, no. 2, pp. 509-522, Feb. 2016 